\title{
SPATIAL VECTORS AND THE ANGLES THEY ENCLOSE
}

\author{
BY \\ E. J. FISCHMANN \\ From the Cardiology Department, Green Lane Hospital, Auckland, New Zealand \\ Received March 1, 1955
}

The spatial vector and loop allow a more concise description of the heart's total electromotive force at an instant, and throughout the cardiac cycle, than the conventional electrocardiogram and the three-plane vectorcardiogram.

The magnitude of spatial vectors and the angles enclosed by these vectors are independent of cardiac position. Their relationship to the cardiac forces is therefore relatively simple. This suggests a promising new approach to the hitherto unrewarding quantitative analysis of the electrocardiogram.

With all lead-systems now in common use, the accuracy of quantitative measurement is limited by the errors inherent in Einthoven's hypothesis. These errors are augmented especially with regard to the sagittal component, where vectors are by-products of unipolar chest lead recording as with the ingenious mechanical models of Langner (1952), Urschel and Denton (1952), and Simonson and Ross (1953), and with the electronic method of Donzelot et al. (1950). The phaseshift error, introduced by recording leads separately with a single-channel instrument, is regarded as significant by Holzmann (1945, p. 58) and Burch et al. (1953), and as unimportant by Goldberger (1953).

In the present study the frontal lead-pair Y, X and the coronal pair Z, X of the "cube " lead arrangement, were recorded with a two-channel electrocardiograph. The technique of recording has been described (Fischmann and Brown, 1954). Spatial vectors have been determined by correlating frontal and coronal lead-pairs. Lead X, which appears in both lead-pairs, is used to identify synchronous points in the two records.

\section{Non-QuANTITATIVE Method of ANALYsis}

The orientation of the spatial QRS loop is that of its longest vector. In the scalar component lead record, the peak of the greatest QRS deflection (Qy in Fig. 2A; $\mathrm{Q} z$ in Fig. 2B; $\mathrm{R}^{\prime} y$ in Fig. 2C) marks the instant corresponding to this vector (Fischmann and Brown, 1954). Consideration of $x, y$, and $z$ (voltages in leads $\mathrm{X}, \mathrm{Y}$, and $\mathrm{Z}$ ) at this instant, will give the general orientation of the longest spatial QRS vector. As shown in Fig. 1, positive deflections in lead X mean left-, in lead Y head-, in lead $Z$ forward orientation of the instantaneous vector. Thus for instance in Fig. 2A the longest QRS vector is foot- $(\mathrm{Q} y)$, left- $(\mathrm{R} x)$, and forward $(\mathrm{Rz})$ orientated. The order of enumeration indicates the relative size of the three components. The small initial $r$ or $q$, and terminal $\mathbf{r}^{\prime}$ or $\mathbf{s}$ deflections in leads $\mathrm{X}, \mathrm{Y}$, and $\mathrm{Z}$ can be similarly employed to determine the spatial orientation of initial and terminal vectors; $x, y, z$ corresponding to the peak of the greatest $\mathrm{T}$ wave, the orientation of the $\mathrm{T}$ loop. Records from a normal subject, and from subjects with left and right ventricular hypertrophy, are shown in Fig. 2, and analysed in the legend. 


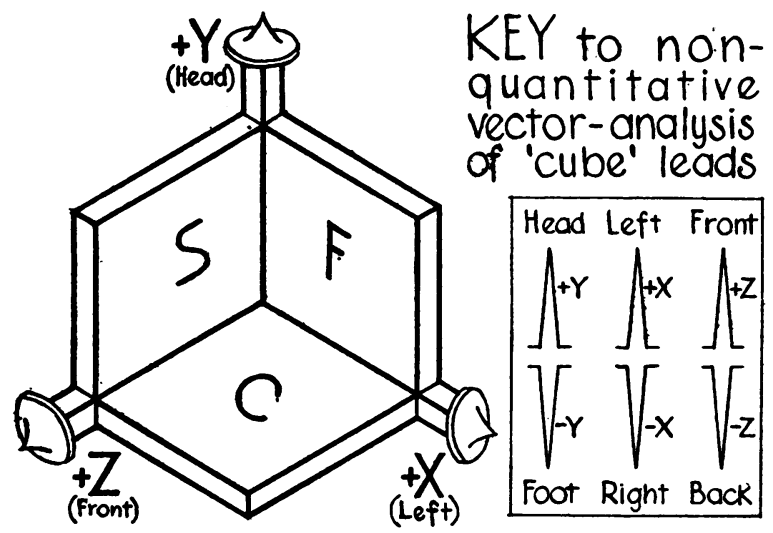

A

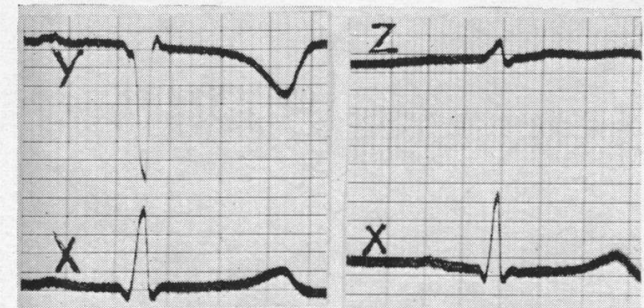

B
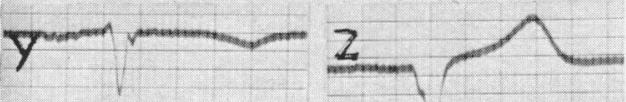

C
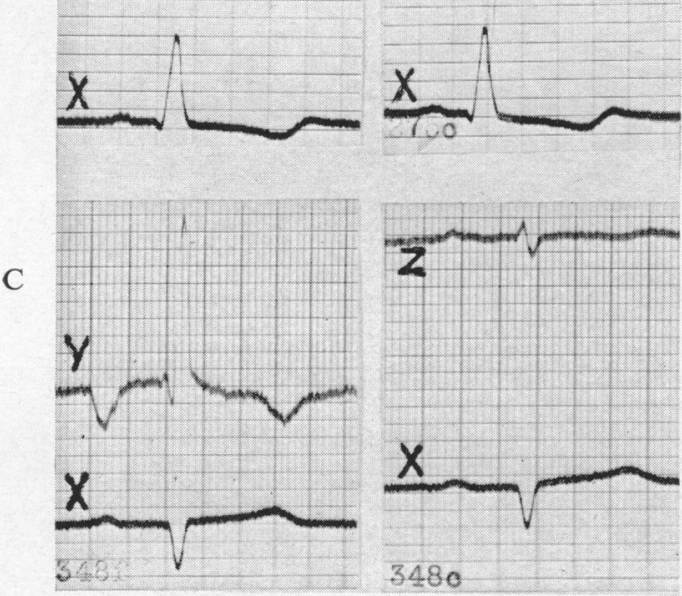

Frontal

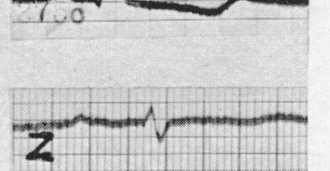

\section{0}

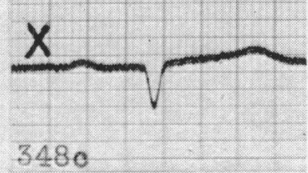

FIG. 1.-Polarity of the three leads $(Y, X, Z)$ of the "cube" lead arrangement. F frontal, C coronal, $\mathbf{S}$ sagittal planes, defined by the axes of these leads. Left anterior oblique view. The three leads have a common zero point (the right lumbar electrode). Vectors directed away from this point give a positive deflection in the corresponding lead. Thus (inset) positive deflections in leads $Y, X$, and $Z$ mean headward, left, and forward directed vectors respectively; and negative deflections mean opposite vector directions as shown.

FIG. 2.-The spatial orientation of $Q R S$ and $T$ loop, i.e. of longest $Q R S$ and $T$ vector, by simple inspection of frontal and coronal leadpairs. Non-quantitative method. $X$ transverse, $Y$ caudo-cranial, $Z$ sagittal lead. Time marks $1 / 10$ and $1 / 50$ sec. Calibration $1 \mathrm{mV}$ $=1.5 \mathrm{~cm}$. (A) Normal heart. The nadir of greatest deflection $\mathrm{Q} y$ (footward) marks the instant of the longest QRS vector. Synchronously with the latter, lead $X$ shows positive (leftward), lead Z slightly negative (backward) voltage. The longest QRS vector is thus footward, left, and backward orientated; the order of enumeration shows the relative size of the three components. $\mathrm{T}(-y, x, z)$ is footward, left, and forward. (B) Left ventricular hypertrophy. Synchronously with greatest deflection $\mathbf{Q} z$ (backward), lead $\mathbf{X}$ shows positive (left), lead Y negative (footward) voltage: backward, left, footward orientated QRS loop. T $(z,-x,-y)$ is forward, right, footward. (C) Right ventricular hypertrophy, right bundle branch block. Synchronously with peak of greatest deflection $\mathbf{R}^{\prime} y$ (headward), lead X shows negative (right), lead $\mathrm{Z}$ also negative (backward) voltage: headward, right, backward QRS loop. $T$

$(-y, x, z)$ is footward, left, forward.

\section{Quantitative Method of Analysis}

Vector Summation of Three Components. At any instant the voltages $x, y$, and $z$ in leads $\mathrm{X}$, $\mathrm{Y}$, and $\mathrm{Z}$ are transverse, caudo-cranial, and sagittal components of the spatial cardiac vector Esp.

$$
\mathrm{Esp}=x+y+z
$$

The vector sum of $x, y$, and $z$ can be obtained by setting $y$ off from the end of $x$, then setting $z$ off from the end of $y$, and connecting free onset with free end (Henrici and Turner, 1903; Rutherford, 
1951). In keeping with the "law of commutation," components may be drawn in any desired order. Thus in Fig. 3A $x$ follows $z$, and $y$ follows $x$. It is not possible to perform the summation of three non-coplanar components, without transformation into coplanar pairs. Transformation is easily achieved if in equation (1) $x+y$ is replaced by Ef, or $x+z$ by Ec, or $y+z$ by Es; where Ef, Ec, and Es are frontal, coronal, and sagittal instantaneous vectors. Equation (1) can then be re-written as follows:

$$
\mathrm{Esp}=\mathrm{Ef}+z=\mathrm{Ec}+y=\mathrm{Es}+x \quad \text {. . . . . . . . . . . }
$$

This is to say that at any instant the spatial cardiac vector is the vector sum of a plane vector and of the voltage in the non-coplanar component lead. The sum of $\mathrm{Ec}$ and $y$ is used in the present study. Each spatial vector is determined in two stages, expressed by the equations $x+z=\mathrm{Ec}$ and $\mathrm{Ec}+y$ $=$ Esp. All terms of equation $x+z=\mathrm{Ec}$ are in the coronal plane (Fig. 3B); those of equation $\mathrm{Ec}+y=\mathrm{Esp}$, in a plane which is perpendicular to the coronal and intersects the latter plane in a line corresponding to the coronal vector (Fig. 3C).

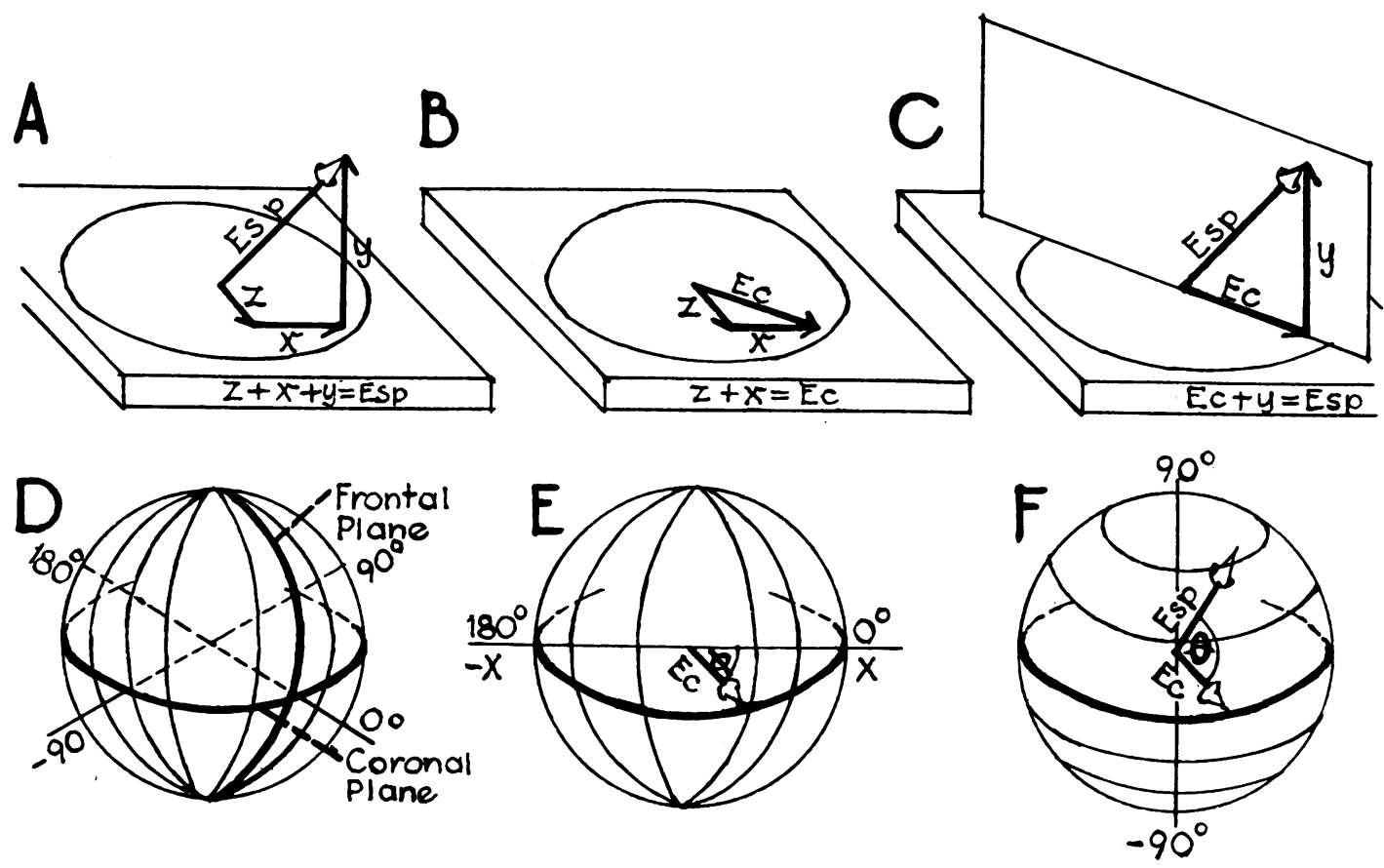

Fig. 3.-The vector summation of three components. Ec coronal, Esp spatial vector; $x, y, z$ instantaneous voltages in leads $\mathrm{X}, \mathrm{Y}, \mathrm{Z} ; \phi$ longitude, $\theta$ latitude angle. (A) Esp as the vector sum $z+x+y: x$ set off from end of $z$; $y$ set off from end of $x$; Esp connects free onset with free end. (B and C) Esp obtained by two coplanar summations: (B) $x$ set off from end of $z$, Ec connects free onset with free end. (C) $y$ set off from end of Ec, Esp connects free onset with free end. (D) Spatial coordinate system. (E) Ec and the X axis enclose longitude angle. (F) Esp and Ec enclose latitude angle.

Spatial Coordinate System. The direction of frontal, coronal, and sagittal plane vectors can be stated in terms of a circular scale, such as Einthoven's, if all vectors in the plane are drawn to commence from the centre of the scale. In the present study this type of representation has been extended as follows: the direction of any spatial vector commencing at the centre of a sphere can be indicated by marking a point on the sphere's surface. To be able to state the position of such points, a coordinate network will have to be drawn on the sphere. The earth's longitude-latitude network was chosen for this purpose (Fig. 3D). It has the advantage of being well known and easily visualized. The coronal plane was laid through the equator, the frontal plane through the $0^{\circ}$ longitude great circle, of the globe. "Viewing" the coronal plane from below, the negative 
angles of the Einthoven scale in that plane are disposed anteriorly. Longitude, ranging from $0^{\circ}$ to $180^{\circ}$, was therefore called negative on the anterior, positive on the posterior hemisphere. Latitude ranged from $0^{\circ}$ at the equatorial circle, headward to $90^{\circ}$, and footward to $-90^{\circ}$. The angle $\phi$, enclosed by Ec and the $\mathrm{X}$ axis, is identical with the longitude (Fig. 3E), and the angle $\theta$, enclosed by Ec and Esp with the latitude (Fig. 3F), of the spatial vector.

Construction of Spatial Vectors. The two vector summations $z+x$ and Ec $+y$ can be carried out on the ECG strip (Fig. 4). As an alternative method a biaxial chart (Fischmann and Brown, 1954 (Fig. 2)) may be used for the summation of $x$ and $y$; whilst Fig. 5 of the present paper was employed for the summation of Ec and $y$.

The Angle Enclosed by Two Spatial Vectors. The significance of the QRS-T angle was shown by Grant $e$ t al. (1951), further studied on mechanical vector models by Langner (1952), Urschel and Denton (1952), and Simonson and Ross (1953) and, with the aid of trigonometric tables by

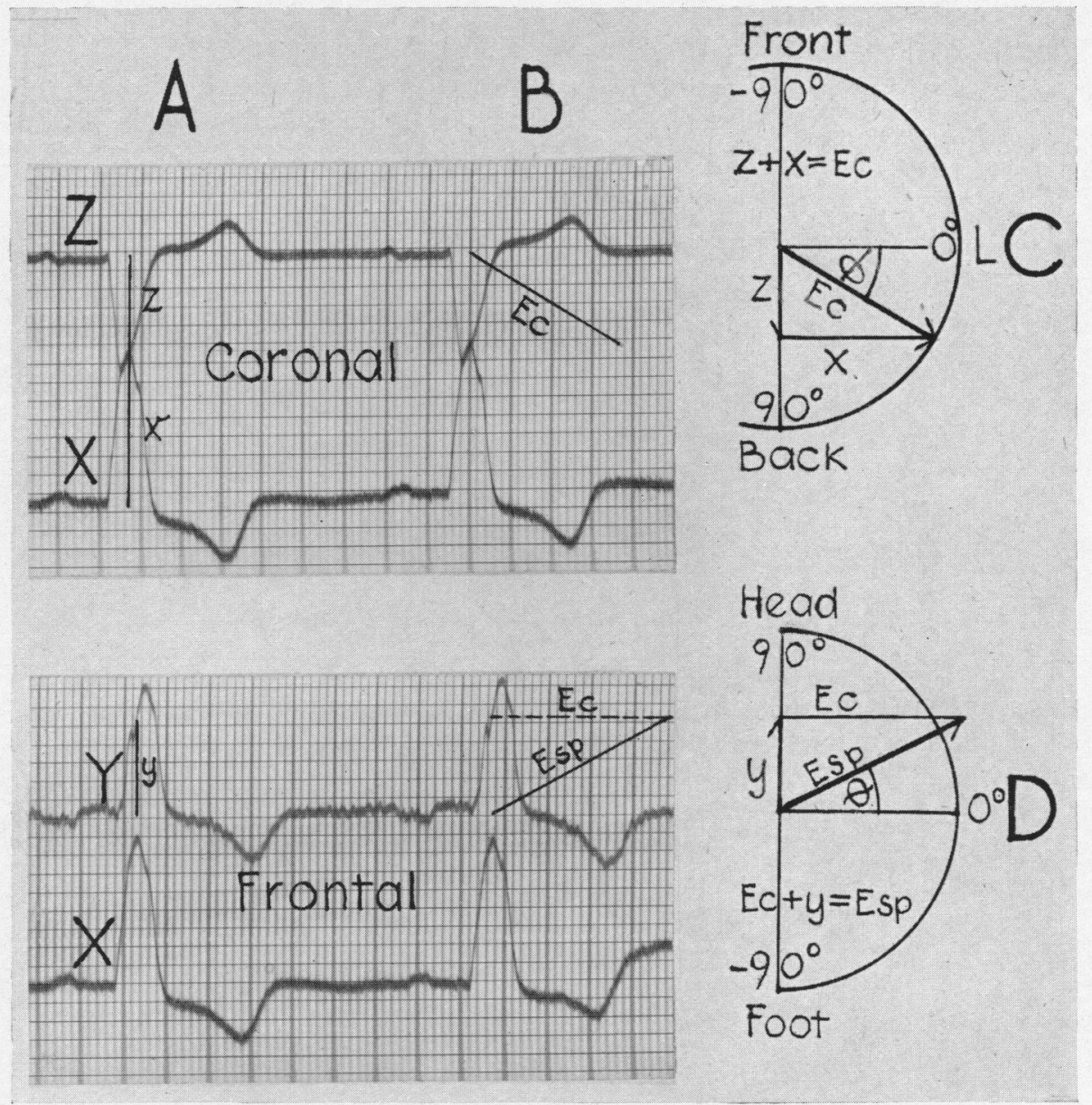

Fig. 4.-Construction of a spatial instantaneous vector on the ECG strip. Quantitative method. Abbreviations as Fig. 3. (A) $x, y$, and $z$ at an instant corresponding to peak of $\mathrm{R} x$. (B) On the coronal lead strip $x$ is set off from the end of $z$. Ec connects free onset with free end. Ec is then transferred to frontal lead-pair, and set off from the end of $y$. Esp connects free onset with free end. Longitude is the angle enclosed by Ec and the horizontal; latitude the angle enclosed by Esp and the horizontal. (C) and (D) Graphic representation of these operations. Time marks $1 / 10$ and 1/50 sec. Calibration $1 \mathrm{mV}=1 \cdot 5 \mathrm{~cm}$. 
FIG. 5.-Biaxial chart for the summation of the coronal vector Ec, and the non-coplanar lead voltage $y$ (modified after Wenkebach and Winterberg). The concentric semicircles divide the radii of the figure, including the vertical axis $Y$ (representing the axis of the caudo-cranial lead) and the horizontal axis (representing the coronal vector), into ECG amplitude $\mathrm{mm}$.

The terminus of Ec is marked on the horizontal axis. The vertical line passing through the terminus of Ec is then followed upward if positive, and downward if negative, to a point corresponding to the magnitude of $y$; this point is the terminus of the spatial vector Esp. The magnitude of Esp is read on the scale formed by the concentric circles; the latitude angle read at the border of the chart.

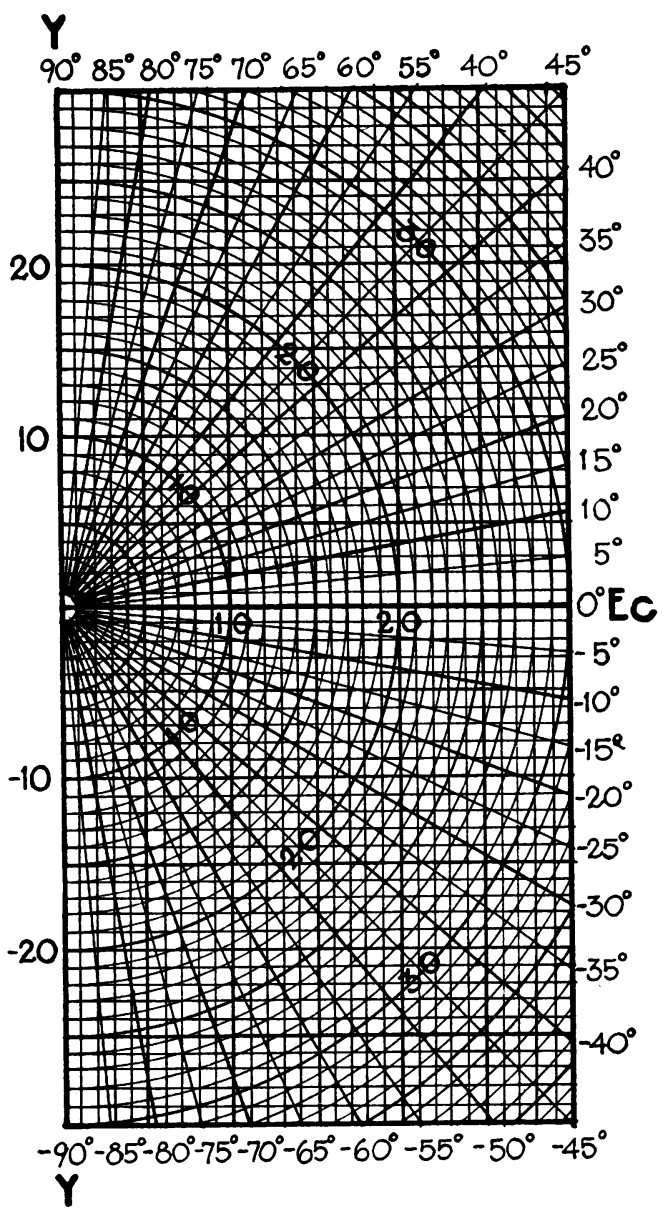

Helm and Fowler (1953). The angle enclosed by two vectors can be measured rapidly and simply, on a "blackboard" terrestrial globe showing the longitude-latitude network without the earth's map. Two chalk marks, corresponding to the two vectors, are made on the globe (Fig. 6A). The

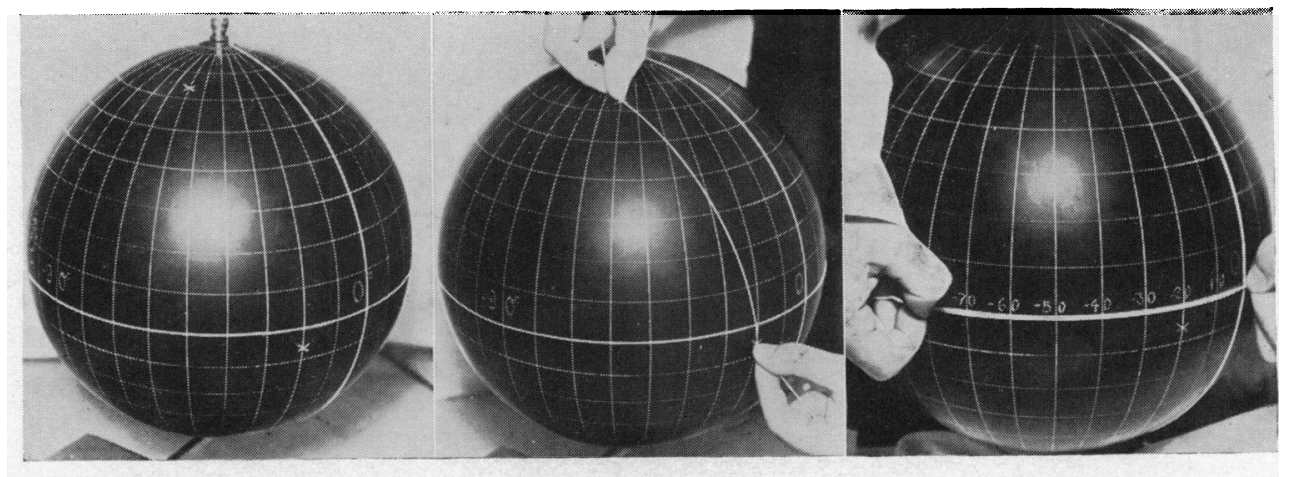

FIG. 6.-Measurement of the angle enclosed by two spatial vectors. (A) The two chalk crosses on the blackboard globe correspond to two spatial vectors. (B) The shortest distance between the two marks is measured with a cotton thread. (C) The angle enclosed by the two vectors is read after placing the thread along the equator of the globe. 
shortest distance between the two marks on the globe's surface is then measured with a cotton thread (Fig. 6B). If the thread is placed on the equator, the angle enclosed by the two vectors can be directly read (Fig. 6C).

Single-curve Vectorcardiogram. If points corresponding to consecutive vectors of a cardiac cycle were marked on the surface of the globe, the curve connecting these points would indicate the direction but not the magnitude of instantaneous spatial vectors throughout the cardiac cycle. Such a curve can be transferred onto a flat sheet of paper, as maps are "projected" from the terrestrial globe onto a sheet (Steers, 1949). Thus one curve would supply the data (concerning spatial vector orientation) contained in the three loops of the cathode-ray vectorcardiogram. Such normal and abnormal curves are being studied at present at this laboratory as are tentatively called "single-curve vectorcardiograms."

\section{Discussion}

Using readily available recording apparatus the non-quantitative method of analysis described, yields certain characteristics of the spatial vector loop by simple inspection, as does the three-plane vectorcardiogram. This method may be applied to clinical problems as will be described in later studies.

Construction on the electrocardiographic strip or on two biaxial charts was used for quantitative determination of the magnitude and orientation of spatial vectors. A " blackboard globe" model was used only for the measurement of angles between vectors. Comparison with published descriptions of vector models shows the relative ease of using the globe. The latter is commercially available, thus no special apparatus need be built; instead of manipulating "vector rods" or pointers, two chalk marks are made; a large number of vectors can be simultaneously represented.

The coronal plane is " viewed" from above in most published work (Committee of American Heart Association, 1954); from below by Grant and Estes (1951), Shillingford and Brigden (1951), and with the present method. Reasons for choosing one of the two possible "points of view" are not given in most published studies. It was felt that with the present method, as scalar leads were used, " consistency in convention of sign" (Robertson, 1951) should be observed. Leads of the "cube" lead arrangement so recorded, resemble in their patterns conventional leads with parallel axes, facilitating correlation. Forward directed vectors give upward deflections in conventional leads with sagittal or near-sagittal axes (V1, 2, 3), also in " consistently" recorded lead $\mathrm{Z}$. This is to say that these leads present the coronal plane as seen from below.

\section{SUMMARY}

Methods for the determination of spatial cardiac vectors, from leads of the " cube " reference system, have been described. A two-channel scalar electrocardiograph was used to record a frontal lead-pair (caudo-cranial and transverse lead), and a coronal lead-pair (sagittal and transverse lead).

Spatial vectors and loops can be described in a non-quantitative fashion, from simple inspection of the two lead-pairs. A method for the quantitative determination of the magnitude and direction of spatial vectors, on the electrocardiographic strip or on two biaxial charts, is suggested. The longitude-latitude network of the terrestrial globe is used as a spatial coordinate system, to define the direction of spatial vectors. A terrestrial "blackboard globe" is used as a vector model, to determine the angle enclosed by two spatial vectors.

A new principle of representing all spatial vectors of a cardiac cycle by one symbol, the " singlecurve vectorcardiogram," is discussed.

I wish to thank the Physicians to Green Lane Hospital for their permission to investigate patients in their care; the Medical Superintendent and the Board for their cooperation; and Drs. D. Brown and K. Kreielsheimer, Associate Professors of Physics, Auckland University College, for their advice concerning the mathematical aspects of this study. 


\section{REFERENCES}

Burch, G. E., Abildskov, J. A., and Cronvich, J. A. (1953). Spatial Vectorcardiography. London.

Committee on Electrocardiography, American Heart Association (1954). Circulation, $10,564$.

Donzelot, E. Milovanovich, J. B., and Kaufmann, H. (1950). Etudes Pratiques de Vectographie. L'Expansion Scientifique Francaise.

Fischmann, E. J., and Brown, D. (1954). Brit. Heart J., 16, 351.

Goldberger, E. (1953). Unipolar Lead Electrocardiography and Vectorcardiography. Philadelphia.

Grant, R. P., Estes, E. H., and Doyle, J. T. (1951). Circulation, 3, 182.

Helm, R. A., and Fowler, N. O. (1953). Amer. Heart J., 45, 835.

Henricic, O., and Turner, G. C. (1903). Vectors and Rotors. London.

Holzmann, M. (1945). Klinische Electrocardiographie. Zurich.

Langner, P. H. (1952). Amer. Heart J., 44, 373.

Robertson, D. (1951). Amer. Heart J., 42, 412.

Rutherford, D. E. (1951). Vector Methods. Edinburgh.

Shillingford, J., and Brigden, W. (1951). Brit. Heart J., 13, 233.

Simonson, E., and Ross, J. (1953). Circulation, 7, 403.

Steers, J. A. (1949). The Study of Map Projections. 7th ed. London.

Urschel, D. L., and Denton, C. A. (1952). Amer. Heart J., 44, 372.

Wenkebach and Winterberg (1927). Quoted from Holzmann, p. 60. 